\title{
4D-STEM analysis of an amorphous-crystalline polymer blend: combined nanocrystalline and RDF mapping
}

Jennifer Donohue ${ }^{1}$, Karen C Bustillo ${ }^{2}$, Steven E Zeltmann ${ }^{1}$, Colin Ophus ${ }^{3}$, Benjamin Savitzky ${ }^{3}$, Mary Ann Jones $^{4}$, Gregory F Meyers ${ }^{4}$ and Andrew Minor ${ }^{5}$

${ }^{1}$ University of California, Berkeley, United States, ${ }^{2}$ Lawrence Berkeley National Laboratory, United States, ${ }^{3}$ Lawrence Berkeley National Laboratory, California, United States, ${ }^{4}$ DOW Chemical Company, United States, ${ }^{5}$ UC Berkeley, Berkeley, California, United States

Nanoscale morphology of multiphase polymer systems and blends, particularly at and near interfaces, is critical to understanding interphase adhesion mechanisms and their impact on bulk mechanical properties ${ }^{[1-2]}$. To visualize this morphology over a representative sampling, a fast high-resolution technique for mapping both crystalline and amorphous domains with a large field of view is needed. Transmission electron microscopy (TEM) offers the high resolution needed. However, the application of TEM to polymers is severely limited by the electron beam sensitivity, low scattering intensity from low-Z elements, and difficulty in differentiating polymers which often have close chemical and structural makeup. To circumvent this traditionally, a heavy metal stain is used to introduce artificial contrast, but this contrast can be very difficult to interpret as it provides little structural information and often results in artifacts. Four-dimensional scanning TEM (4D-STEM) is an emerging technique with high spatial resolution over a relatively large sample area which provides a wealth of structural information ${ }^{[3]}$. However, beam damage has limited its applications for soft materials and polymeric blends ${ }^{[1-2]}$. Here we explore the use of high-speed detectors, small condenser apertures, and high throughput processing software for 4D-STEM characterization of both the amorphous and crystalline regions of polymer blends. Specifically, we demonstrate a low-dose 4D-STEM technique for visualizing nanostructure in extremely beam sensitive semicrystalline isotactic polypropylene and ethylene-octene copolymer (iPP/EOC) multilayer films.

First, we introduce radial distribution function (RDF) analysis as a method to identify and differentiate the amorphous phases of these two chemically and structurally similar polymers (Figure 1). The derived RDFs for iPP and EOC and the identified peaks match well to literature ${ }^{[4-6]}$ within the resolution of this technique. Here we utilize the peak located at $6.5 \AA$, representative of the repeat unit length of iPP helices ${ }^{[7]}$ to differentiate the two phases producing an amorphous phase map as shown in Figure 1. The lighter region of the map is identified to be iPP, and a clear material interface can be distinguished. By comparison traditional virtual bright field (BF) imaging reveals only contrast from the underlying carbon support, with the material interface is completely obscured. This RDF technique, however, is easily able to reveal the interface with little or no influence from the support.

With the amorphous structure analyzed and the distribution mapped, we present a second technique for identifying and visualizing crystalline domains. A correlative grain growth (CGG) method was developed as a high-throughput technique to identify individual nanoscale crystallites within the materials. CGG uses diffraction patterns with high crystalline diffraction intensity as seeds and grows crystalline domains based on multiplicative correlation of neighboring diffraction patterns. This method is able to map crystallites with very low intensity diffraction, and differentiate neighboring crystallites and even in some cases overlapping crystallites, allowing for statistical analysis of size, location, and shape of individual crystals over a large field of view. The CGG-derived crystalline map is shown in Figure 2 with example diffraction patterns. By comparison, virtual annular dark field and virtual BF images showed no distinct distinguishable crystallites, demonstrating the strength of the CGG method for detecting crystals. 
In summary, we have demonstrated a technique for visualizing the amorphous and crystalline nanoscale structure in a multilayer iPP/EOC film, overcoming beam sensitivity, chemical and structural similarity, and low diffraction contrast limitations.

\section{Acknowledgment}

This work was supported by the Dow University Partnership Initiative Program. Work at the Molecular Foundry at Lawrence Berkeley National Laboratory was supported by the U.S. Department of Energy under Contract \# DE-AC02-05CH11231.
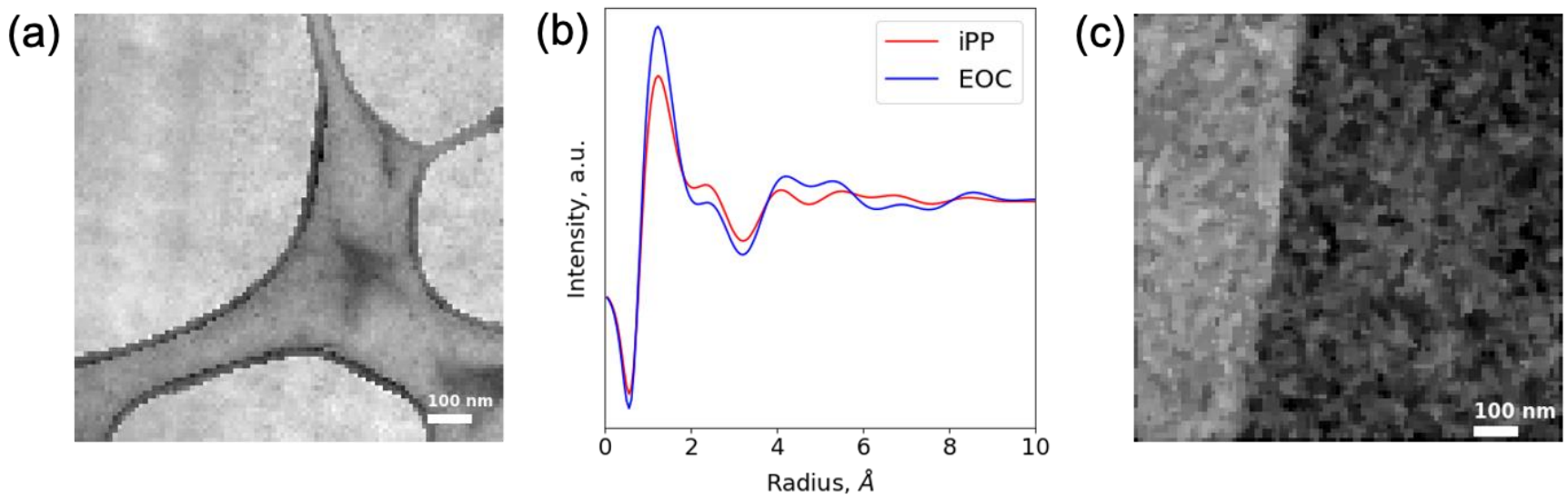

Figure 1. (a) Virtual BF image of iPP/EOC film, (b) derived RDF profiles for iPP and EOC, and (c) RDF derived phase map using 6.5A spacing, bright pixels indicate iPP

(a)

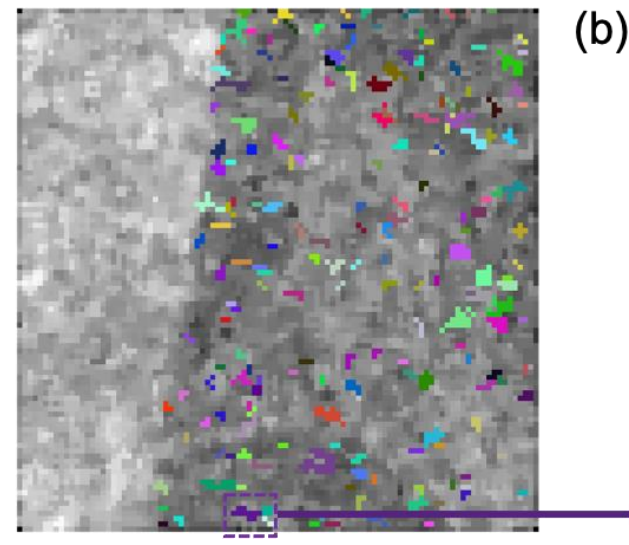

(b)

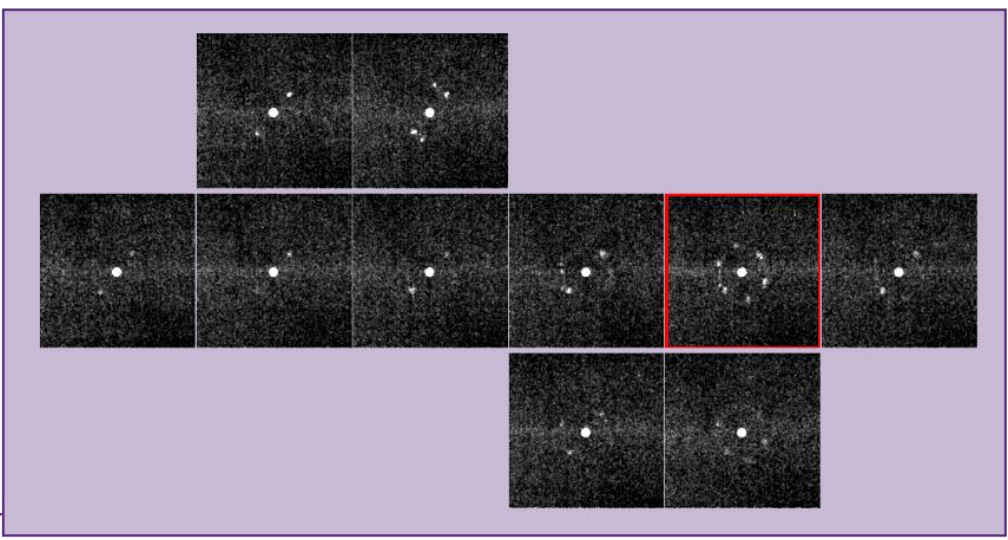

Figure 2. (a) crystallite map showing differentiated crystallites identified using CGG method overlaid on RDF derived phase map, and (b) example of 4D-STEM diffraction patterns assigned to a single crystallite by CGG

\section{References}

[1] Panova, O., Ophus, C., Takacs, C. J., Bustillo, K. C., Balhorn, L., Salleo, A., ... \& Minor, A. M. (2019). Diffraction imaging of nanocrystalline structures in organic semiconductor molecular thin films. Nature materials, 1 . 
[2] Mu, X., Mazilkin, A., Sprau, C., Colsmann, A., \& Kübel, C. (2019). Mapping structure and morphology of amorphous organic thin films by 4D-STEM pair distribution function analysis. Microscopy.

[3] Ophus, C. (2019). Four-Dimensional Scanning Transmission Electron Microscopy (4D-STEM): From Scanning Nanodiffraction to Ptychography and Beyond. Microscopy and Microanalysis, 25(3), 563-582.

[4] Narten, A. H. (1989). Radial distribution of carbon atoms in crystalline and molten polyethylene from xray diffraction. The Journal of chemical physics, 90(10), 5857-5860.

[5] Wang, C. S., \& Yeh, G. S. Y. (1981). Effects of Radiation on the Structure of Polypropylene. Polymer Journal, 13(8), 741-747.

[6]Curro, J. G., Weinhold, J. D., Rajasekaran, J. J., Habenschuss, A., Londono, J. D., \& Honeycutt, J. D. (1997). Intermolecular packing in stereoregular polypropylene liquids: Comparison between theory and X-ray scattering experiments. Macromolecules, 30(20), 6264-6273.

[7] Marigo, A., Martorana, A., Marega, C., \& Zannetti, R. (1987). Radial atomic distribution function and structure models of isotactic polypropylene. Die Makromolekulare Chemie, Rapid Communications, 8(1), 2326. 